\title{
A Review on Digital Image Restoration Process
}

\author{
Sujita Pillai \\ Oriental College of Technology
}

\author{
Sanjay Khadagade \\ Oriental College of Technology
}

\begin{abstract}
Image restoration is the process of restoring degraded images which cannot be taken again or the process of obtaining the image again is costlier. We can restore the images by prior knowledge of the noise or the disturbance that causes the degradation in the image. Image restoration is done in two domains: spatial domain and frequency domain .image can provide an insight for filtering operations. After the filtering, the image is remapped into spatial domain by inverse Fourier transform to obtain the restored image. Restoration efficiency was checked by taking signal to noise ratio (snr) and mean square error(mse) into considerations.
\end{abstract}

\section{Keywords}

Restoration, De-blur, De-convolution, Filtering, Noise.

\section{INTRODUCTION}

The Digital snapshot Processing allows for a wider variety of algorithms to be utilized to the input information and might hinder issues such because the build-up of noise and signal distortion in the course of processing. The giant discipline of Digital snapshot Processing Is gaining quite a few research interest now days. In contemporary years, probably the most lively area in Digital snapshot Processing is of photograph Restoration. It is an subject Underneath Digital image processing where the fashioned photos are restored from the degraded ones. Photograph Restoration is to get better the long-established and sharp picture from a degraded photograph with the aid of utilizing a mathematical mannequin of the blurring method. Right here, we first take an photograph which is referred to as usual photograph. The original photograph is degraded utilizing a degradation operate and by including a noise to it. This degraded image can then be restored utilizing the little knowledge to be had concerning the degradation operate and supply of noise. The method of snapshot t Restoration is split into two phases named as degradation section and restoration segment Each phases are mentioned below:

\section{A MODEL OF IMAGE \\ DEGRADATION}

A Model of image degradation and restoration process the basic unit of a image is called a pixel or image element i.e. the image is divided into very small blocks called pixels. An image can be defined as a two dimensional function I

$I=f(x, y)$

where $\mathrm{x}$ and $\mathrm{y}$ are spatial coordinates. $(\mathrm{x}, \mathrm{y})$ represents a pixel. I is the intensity or grey level value which is the amplitude of $f$ at any point $(x, y)$. If the values of the coordinates (spatial coordinates) and the amplitude are finite and discrete, then it is called digital image. The degraded image $\mathrm{g}(\mathrm{x}, \mathrm{y})$ can be represented as

$$
g(x, y)=h(x, y) * f(x, y)+n(x, y)
$$

an image undeniably is to avoid the need for restoring the image. [11]

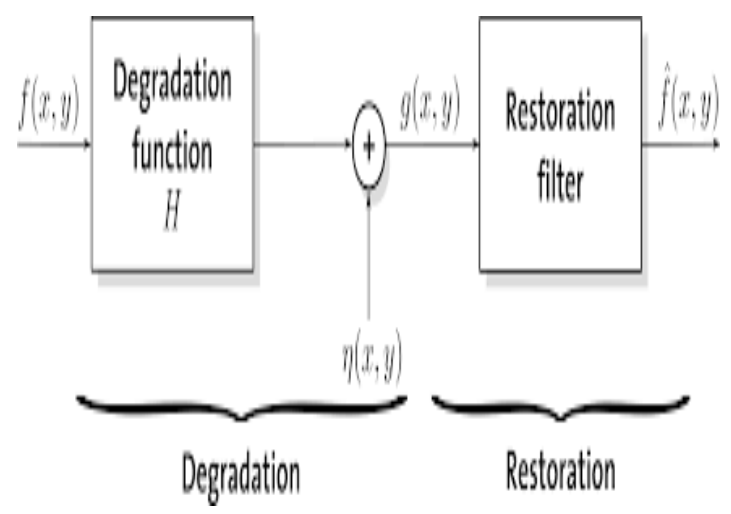

Fig.1 Image Degradation model

\section{DEGRADATION PHASE}

In this section, the fashioned image is degraded with a degradation operate and an additive noise. The consequent snapshot of this segment is a degraded picture. In this phase, the degraded picture is restored utilizing more than a few restoration filters and An estimated image of the original photo is produced as an output. Snapshot Restoration ways will also be divided into two classes [7]:

\section{- Blind \\ - Non Blind}

\subsection{Blind}

Blind Restoration is the one where the blurring operator is unknown; we must make an estimate of the blurring operator after which making use of that estimate we have to de-blur the snapshot.

\subsection{Non Blind}

The Non Blind Restoration is the one wherein the blurring operator is famous; we are able to without problems do away with blur from the degraded picture using the expertise of blurring function. [1]

There is a quantity of procedures available for photograph Restoration. These are Weiner Filtering process (WFT) [3], Regularized scholar's-t probability density perform (PDF).Richardson method (LRT) [2], and Blind image Deconvolution method (BID) [3].

\section{PROPOSED METHOD}

In this section we provide general overview of the proposed technique. The main objective of the proposed blind fusion scheme is to perform improved image fusion in the presence of unknown image degradation. The task of image restoration is often performed prior to and separate from fusion. This solution is suboptimal as the information from the fusion process can often contain relevant information to improve the 
restoration which can in turn provide improved fusion. Here in this dissertation the proposed method for image restoration which is the combination of two different restoration methods to obtain the final restored image?

Restoration techniques are oriented toward modelling the degradation and applying the inverse process in order to recover the original image. The image gets blurred due to the degradation. Blur is of many types but in this thesis work considered the motion blur only.

Mainly the techniques which used in this work are:

- $\quad$ Lucy Richardson fused with Blind Deconvolution [5]

- Lucy Richardson fused with Wiener restoration

- Lucy Richardson fused with regularized filter.

Complete Literature assessment in the subject of photograph Restoration to gift the evaluation of quite a lot of restoration algorithms has been observed.

\section{LITERATURE SURVEY \\ 5.1 Li Yang et.al in 2016}

The blur which is caused by the relative motion between the camera and object scene is called motion-blur. The blur direction and the motion-blurred image are essential for image restoration, which is degraded by the relative motion. And directly affect subsequent image recognition and image analysis. The recently developed Approach based on a fractional directional derivative operator with non-causal is derived. In order to have a grasp of image restoration from motion-blurred photograph, a partial derivative operator is deduced on the basis of fractional derivative in this paper. According to use of real and synthesized motion blur images quantitative and qualitative performance evaluations are carried out. Noise is the sensitive factor of this method. The experiments based on a non-causal fractional partial derivative mask verified that this method provides better immunity to noise in comparison and accuracy of identifying motion blur than the m-ethod based on integer-order differentiation does in particular large blur length

\subsection{Zhang X. F, Ye H, Tian W.F, Chen W.F [2007][5]}

on this paper, a regularized anisotropic diffusion filter was once offered and utilized to revive the DWI. The awarded filtering approach displayed well posedness and excellent maintenance of edges. To assess its effectively in accounting for the Rician noise, the PSNR and MSSIM metrics were used for the primary time. The outcome bought from the unreal and real knowledge proved the easier performance of the offered filters.

\subsection{Mateos, J., Bishop, T.E., Molina, R., Katsaggelos,A.K[2009][9]}

On this paper they gift a brand new Bayesian methodology for the restoration of blurred and noisy photos. Bayesian ways depend on snapshot priors that encapsulate prior photograph abilities and avert the in poor health-posedness of photo restoration issues. They use a spatially various snapshot prior using a gamma-normal hyper prior distribution on the regional precision parameters. The proposed restoration manner is when compared with other photo restoration tactics, demonstrating its expanded performance.
5.4 Ramya, S., Mercy Christial, T[2011][13]

Photo restoration is the approach of getting better the customary picture from the degraded snapshot. Aspire of the undertaking is to restore the blurred/degraded pics utilizing Blind De- convolution algorithm. The principal challenge of photo de-blurring is to de-convolute the degraded image with the PSF that precisely describe the distortion. To begin with, the common picture is degraded making use of the Degradation model. It can be carried out via Gaussian filter which is a low-pass filter used to blur an snapshot. In the edges of the blurred image, the ringing outcomes can also be detected using Canny part Detection approach and then it may be removed before restoration process.admit fast implementation. Experiments are performed on multiple synthetic, grayscale, and color images, and the results demonstrate that the proposed method is effective in blind deblurring.

\subsection{Tomio goto et.al ${ }^{3}$}

In this paper, we propose a blind method that rapidly restores blurred images using local patches. In this method, a portion of the blurred image is used for PSF (point spread function) estimation. In addition, we propose an automatic PSF size calculation algorithm that generates an autocorrelation map (auto map). Experimental results show that our proposed method generates accurate de-blurred images, and processing time is significantly lower than that of the conventional deblurring method.

\subsection{K. Panfilova et.al ${ }^{4}$}

This paper considers the problem of image restoration, in particular, linear blur compensation. To restore image we use Lucy-Richardson iterative method and its modifications. The main disadvantages of the basic method are edge artifacts in the form of horizontal and vertical stripes on the image and lack of information about the optimal number of algorithm iterations. To decrease the negative impact of those disadvantages we propose to extend the image beyond its original borders reducing its brightness to zero at new borders, as well as use an empirical criterion to define the point where the iterative Lucy-Richardson procedure should be finished. The criterion is based on certain analysis of the changes in the image at each iteration. The proposed adaptations resulted in decrease of image distortion by more than $50 \%$ in terms of rms metric, making it possible to estimate the number of iterations required for better performance of the algorithm.

\subsection{Jian-Jiun Ding et.al in 2014 ${ }^{5}$ :}

In image deblurring, it is important to reconstruct images with small error, high perception quality, and less computational time. In this paper, a blurred image reconstruction algorithm, which is a combination of the Richardson-Lucy (RL) deconvolution approach and a pyramid structure, is proposed. The RL approach has good performance in image reconstruction. However, it requires an iterative process, which costs a lot of computation time, and the reconstructed image may suffer from a ringing effect. In the proposed algorithm, we decompose a blurred image from a coarse scale to a fine scale and progressively utilize the RL approach with different number of iterations for each scale. Since the number of iterations is smaller for the large scale part, the computation time can be reduced and the ringing effect caused from details can be avoided. Simulation results show that our proposed algorithm requires less computation time and has good performance in blurred image reconstruction. 


\section{CONCLUSION}

This paper gives a review of different image restoration algorithms. Image restoration is an active research area and various researchers work to improve the efficiency of the different algorithms by developing more efficient algorithms. But primarily image restoration is done mostly using Weiner filter, Richardson-Lucy Blind Deconvolution algorithm, Inverse and Pseudo-inverse filter.

\section{ACKNOWLEDGMENT}

The authors are thankful to Prof. Rahul Dubey -HOD, Dr.N.K Agrawal - IOriental college of technology Bhopal India.

\section{REFERENCES}

[1] Image Restoration From a Single Blurred Photograph Li Yang School of Arts and Communications, Anhui University Hefei 230011, China 2016 3rd International Conference on Information Science and Control Engineering

[2] Fast Weighted Total Variation Regularization Algorithm for Blur Identification and Image Restoration Haiying Liu Member, IEEE, Jason Gu Senior Member, IEEE, Max Q.-H. Meng, Fellow, IEEE Wu-Sheng Lu Life Fellow, IEEE, School of Electrical Engineering and Automation, Qilu University of Technology, Jinan, 250353, China

[3] fast and restoration of blur images based on the local patches tomi goto department of computer science and engineering japan nayoga institute of technology

[4] Linear Blur Compensation in Digital Images Using LucyRichardson MethodK. Panfilova\#1, S. Umnyashkin\#2 Dept. of Higher mathematics National Research University of Electronic Technology Moscow, Zelenograd, Russia

[5] Image Deblurring Using a Pyramid-Based RichardsonLucy Algorithm Jian-Jiun Ding1, Wei-De Chang2, Yu Chen3, Szu-Wei Fu4 Graduate Institution of
Communication Engineering National Taiwan University Taipei, Taiwan

[6] David L. Donoho, "De-noising by soft-thresholding,Dept of Statistics, Stanford University, 1992

[7] Hiroko Furuya and Shintaro Eda, "Image Restoration via Wiener Filtering in the requency Domain",

[8] Hui Li, B.S. Manjunath, Sanjit K. Mitra H. Li, B. S. Manjunath and S. K. Mitra, Multisensor Image Fusion Using the Wavelet Transform, Proc. first International conference on image processing, ICIP 94, Austin, Texas, Vol. I, Pages 51-55, Nov 1994.

[9] Investigations of Image Fusion, Lehigh University.

[10] Jain Anil K.,'Fundamentals of Digital Image Processing”, Davis:Prentice-Hall ofIndia, 2000.

[11] Kundur, D. and D. Hatzinakos, "Blind Image Deconvolution", IEEE SignalProcessing Magazine, vol. 13 (3), pp. 43-64, May 1996.

[12]Kaur. A. Chopra. V, "A Comparative Study and Analysis of Image Restoration Techniques Using Different Images Formats", "International Journal for Science and Emerging Technologies with Latest Trends" 2(1): 7-14 (2012).

[13] L.Prasad and S. S. Iyengar, Wavelet Analysis with Applications to Image Processing. Boca Raton, FL: CRC Press LLC, 1997, pp.101-115.

[14] Mandelbrot, B., and Wallis, J., "Noah, Joseph and operational hydrology," Water Resources Research 4, 909-918, 1968.

[15] M.A.Joshi, Digital Image Processing - An Algorithmic Approach, Professors and Head, Department of Telecommunications, College of Engineering, Pune,Prentice Hall of India Private Limited, New Delhi, 2007. 